\title{
Psychosocial interventions in a case of fibrodysplasia ossificans progressiva: A case report
}

\author{
Manisha Jha, Ruchi Varma Shanker, Anand Kumar, \\ Tej Bahadur Singh
}

\begin{abstract}
Introduction: The developmental needs and challenges of children with chronic illness are different from those of a normal childhood. Moreover, rare disease with no effective treatment option and poor prognosis is devastating for parents also. Case Report: This case report aims to present guided sustained adaptation of a child from India who was diagnosed as a case of fibrodysplasia ossificans progressiva. The child and her parents were referred for psychological intervention in view of rare genetic disease to facilitate positive health. A detailed assessment and psychological intervention was done by trained clinical psychologist. Conclusion: The case report highlighted that caring relationship, formulation of positive meanings about the illness and an opportunity to seek help along with promoting the strengths of the child while she was undergoing a negative experience facilitated child's capacity for innovative survival and ability to resist adversities.
\end{abstract}

Keywords: Fibrodysplasia ossificans progressiva (FOP), Well being, Psychological intervention

Manisha Jha ${ }^{1}$, Ruchi Varma Shanker ${ }^{1}$, Anand Kumar $^{2}$, Tej Bahadur Singh ${ }^{3}$

Affiliations: ${ }^{1}$ Clinical Psychologist, Institute: Institute of Human Behaviour and Allied Sciences, Delhi, India; 2Occupational Therapist, Institute: Institute of Human Behaviour and Allied Sciences, Delhi, India; ${ }^{3}$ Professor in Clinical Psychology, Institute: Institute of Human Behaviour and Allied Sciences, Delhi, India.

Corresponding Author: Dr. Manisha Jha, Clinical Psychologist, Institute of Human Behaviour and Allied Sciences, Delhi -110095, India; Ph: + 91 9868396887, 9891443764; Email: manishajha@hotmail.com

Received: 12 July 2012

Accepted: 17 August 2012

Published: 01 February 2013
Jha M, Shanker RV, Kumar A, Singh TB. Psychosocial interventions in a case of fibrodysplasia ossificans progressiva: A case report. International Journal of Case Reports and Images 2013;4(2):115-119.

$$
* * * * * * * * *
$$

doi:10.5348/ijcri-2013-02-274-CR-8

\section{INTRODUCTION}

Fibrodysplasia ossificans progressiva (FOP) is a rare and disabling genetic condition characterized by congenital malformations of the great toes and progressive heterotopic ossification in specific anatomic patterns. The condition was first reported in the $17^{\text {th }}$ century by Patin, a French physician, who described a woman who "turned into wood". The prevalence of FOP has been estimated at 1/1.64 million persons. Fewer than 200 cases have been described worldwide. FOP is more common in females than in males. FOP is an autosomal dominant condition, but most cases are sporadic. Recently, genetic analysis revealed that the FOP gene is located on chromosome 4 and mutation in this gene causes an over expression of a bone morphogenetic protein (BMP4) in almost all sporadic and familial cases of classic FOP. Minor trauma can trigger proliferation of connective tissue (muscles, ligaments and tendons) resulting clinically as painful swelling of the muscles and connective tissue. This swelling subsides, then after approximately six months or more, heterotrophic ossification starts at some sites in which extra bone formation occurs outside the skeleton. The disease generally presents itself in childhood between 2-6 years and progressively disables the body as the new bones forms bridges and ribbon like structures across the joints and creates immobility. Since no effective medical treatment still exist, most of the patients become bedridden by the time they are in 
fourth decade. This case report aims to present guided sustained adaptation of a child from India who was diagnosed as a case of fibrodysplasia ossificans progressiva. The child and her parents were referred for psychological intervention in view of rare genetic disease to facilitate positive health. Parents offered consent for scientific publication, expecting to receive informal support regarding the disease through this medium. They received psychological intervention for about two years along with continued occupational therapy and physiotherapy. The patient was quite compliant for the therapeutic sessions considering the preventive nature of the therapies.

\section{CASE REPORT}

Patient R was an eight-year-old child studying in 3rd standard. She was third in birth order among her four siblings and belonged to a family with middle socioeconomic status. She presented with a history of spontaneous flare ups (painful and highly inflammatory soft tissue swellings over her body, specifically in right elbow and dorsal surface of medial border and inferior angle of scapula). Flare ups marked the generation of new bone, although not every flare up ends in completion of process. Progressive restrictions in movements of cervical spine, right knee and both elbows were present since two years of age. Joint contractures were present due to bony ankylosis. She had progressive limitation of movement following pain and tumour like growth at the multiple sites (Figure 1). At the time of presentation she had multiple contractures and used compensatory techniques to perform her daily activities. Radiological investigations (X-rays, bone scans) showed microdactyly of big toe (Figures 2 and 3), hallux valgus and ossifications at various sites in body (scapular region and both elbows, more in right elbow). Investigations revealed a normal EMG and normal MRI brain study, however, computed tomography (CT) scan of head showed possible basal ganglia calcification. There were no other neurological deficits. She was diagnosed as a case of fibrodysplasia ossificans progressiva and was advised non- steroidal anti-inflammatory drugs (NSAID) only. A detailed assessment by trained professional was done to assess the current level of cognitive and emotional functioning of the child and family functioning in terms of stress and coping pattern of parents in relation to a chronic illness. This included assessment and intervention by a clinical psychologist and occupational therapist respectively. Her assessment of current functioning revealed inability to use upper and lower limbs properly due to limited movement, inability to sit and she needed physical help to perform self care activities. The patient used log like pattern for movement as there was severe limitation of range of motion (ROM) in all the axial joints. While walking she had fair balance but had a tendency to fall more on the front. Her fine motor prehension was still relatively spared, thus she could use a pen, pencil and computers for academic purposes. She

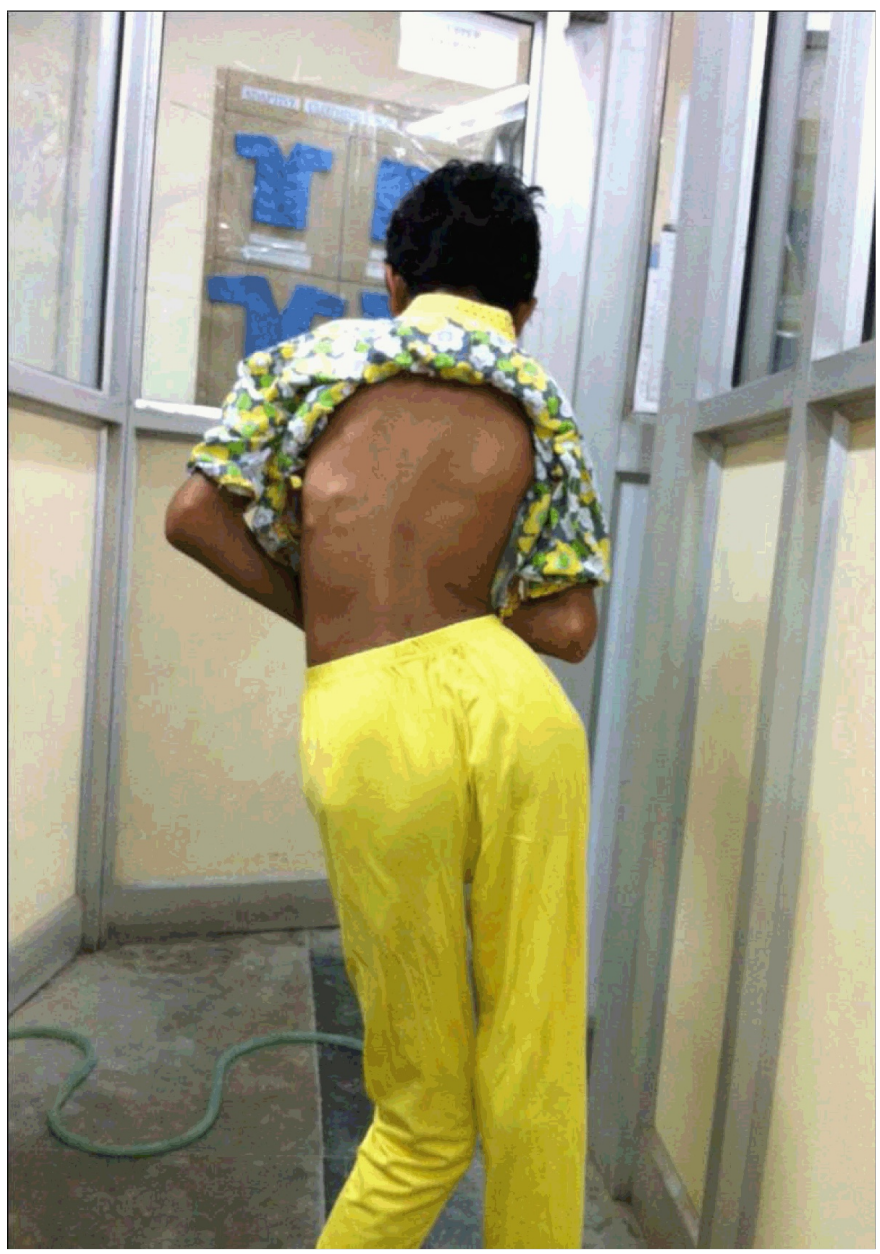

Figure 1: The patient showed spinal deformity in the form of scoliosis. Multiple small ossifications are seen along the inferior angle of left scapula.

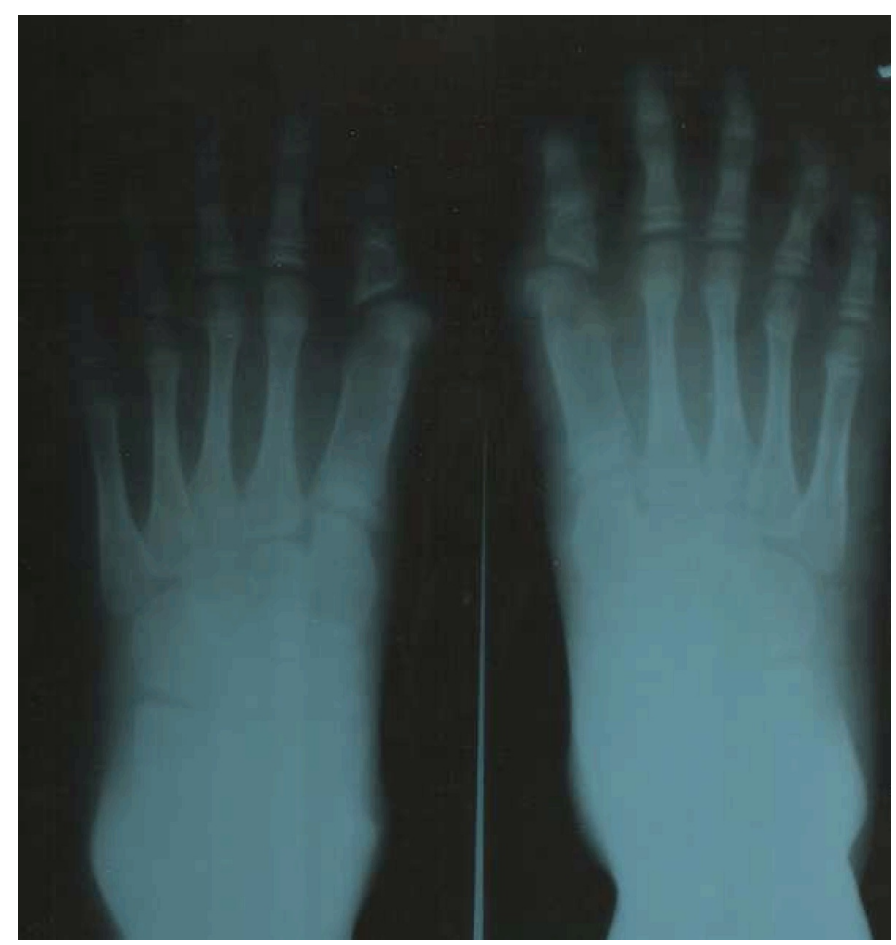

Figure 2: X-ray showing malformation (microdactyly) of big toe. 


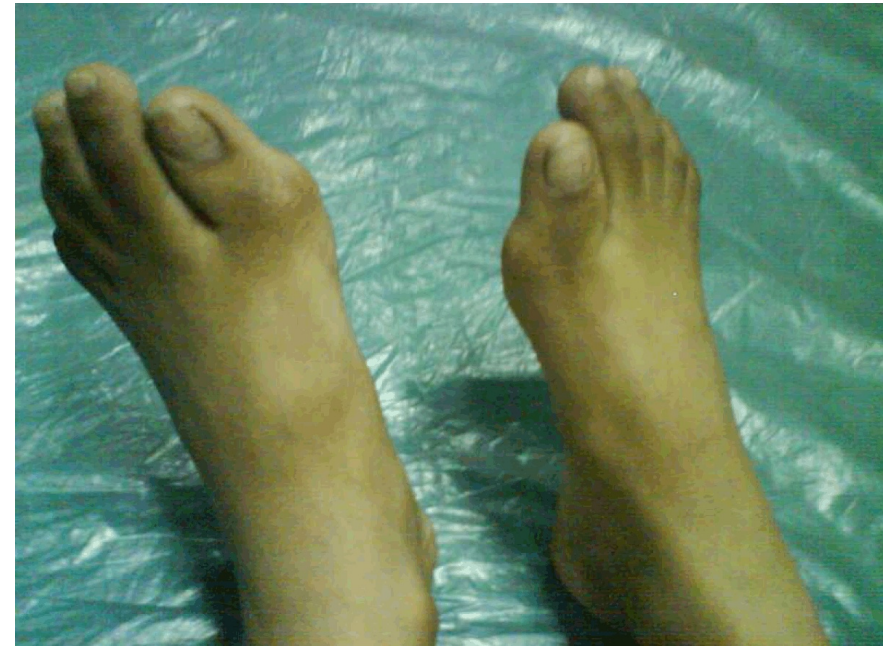

Figure 3: Characteristic feature of fibrodysplasia ossificans progresiva: malformation (microdactyly) of big toe.

had a fair handwriting and reported little difficulties in studies, at school. She attended school regularly. Some modification in her sitting arrangement had to be done at school considering her difficulty to sit on chair. The child did not feel herself to be different from other children in any way and could maintain her friendships.

The psychological tests administered included WISC IIIR [1], Luria Nebraska Neuropsychological Battery for Children [2], House Tree Person Test [3], Rosenzwig Self Esteem scale [4], Children Depression Index [5], Parental Stress Index [6] and Ways of Coping Questionnaire [7]. Since the intervention targeted a range of developmental outcomes, which more closely represent resilience, the General Well Being 20 test [8] was used which measures the impact on a variety of psycho-social constructs. One factor measures the level of occurrence of positive psychological well-being during the past month (or other time frame), and the other factor measures the level of occurrence of psychological distress during the same time frame. Findings of cognitive functions suggested average intelligence with an I.Q. of 101. On assessment, deficits in neuropsychological functioning were noticed in the tactile functions only. Qualitative scoring indicated deficits in the area of motor perseveration, right-left disorientation, stiff motor movements, motor awkwardness and unilateral neglect. Her self-esteem appeared to be moderately high irrespective of her illness status. Assessment on projective test revealed strong need for affiliation and dependence on the peer group. She was quite open to social interaction yet showed control and social tact for her inabilities, with intention to grab opportunity from environment. As far as family unit was concerned; she appeared more assertive, decisive and depicted controlled behaviour which was reflective of a normal secure person. Findings were not suggestive of depressive features. On measure of well-being and distress she scored 32 and 43 respectively, indicating low on well-being and high on distress dimensions. Findings suggested that it could be the parent's perceived or real inability to observe and understand the child's feelings or needs accurately, which somehow restricted them in terms of skill or knowledge regarding child rearing. They often found themselves distancing away from relationships and poor in seeking support. They manifested guilt and unhappy feelings emerging from difficulty to mobilize the psychic and physical energy needed to fulfil parenting responsibility in view of progressively deteriorating health condition. It appeared that their commitment to being ideal parents magnified the source of stress. On the child domain high on demandingness and adaptability may be due to child's physical constraints that made her unable to adjust to changes in her physical or social environment. Coping strategies seen in father was a tendency of wishful thinking to escape or avoid the problems and in accepting responsibility along with poor support seeking. Both the parents were making attempts for planned problem solving and making efforts to create an environment for personal growth.

In psychotherapeutic sessions, information was provided to the child and her parents together, to address the underlying apprehensions, anxieties and concerns considering the nature of illness and process of treatment. At the initial stage of diagnosis, availability of formal sources of social support adequately and on a consistent basis such as advantage of talking with experts, education about the illness and prognosis and information about resources available in meeting with the daily needs of families, improved their understanding and decision making related to disease. The central idea was to reduce parental anxiety which often gets communicated to the child by verbal or nonverbal channels. A few sessions were also devoted to reflect back and restore the belief in self with regard to parental responsibility. This stemmed from the fact that no significant observed change in illness status could be seen in spite of extensive efforts. After the initial impact of the diagnosis of chronic illness was settled, the child and her parents were encouraged to normalize the situation. Supportive therapy with this child was mainly focused on assimilation of her personal asset and basic needs by modifying ways of achieving the set goals. Promoting the strengths of the child while she was undergoing a negative experience was relevant for child's well-being (such as, to seek peer group affiliation, her computer proficiency and interpersonal skills were emphasised to compensate for physical limitation). Thus, improving her social competence, increased comfort with emotional states and creating opportunities together with normally developing peers. For parents problem solving strategies facilitated minimizing the disturbances in day to day activities and conveying the impression of normalcy to others thus, bolstering their coping skills and self-esteem. It seemed intervention was needed to focus on assisting the parents in mobilizing their pride in their child and improving support seeking.

It was focused that parents must maintain a sense of balance in meeting the needs of their ill and healthy children. The central concept was the sense of 
coherence that allowed them to mobilize resources, promote effective coping, and resolve tension in a health promoting manner.

After intervention, score on well-being and distress was 58 and 18, respectively indicating valuable appreciation in her sense of well-being and reduction in distress. Extensive psychological sessions were terminated three years back and presently the child could negotiate well with the significant developmental challenges inspite of some progression in the disease. Family continued therapeutic contact as an when required.

\section{DISCUSSION}

Parents of children and adolescents with newly diagnosed chronic illnesses experience a range of emotions such as guilt, anxiety, shame, or anger [9]. It is more pertinent for rare diseases with lesser known prognosis which could influence child care practices. From the outset of a chronic illness a child also faces potential stressors in the form of threats to bodily integrity and aversive treatment regimens. A change also occurs in the child's interaction with peer group, disruptions in normal family affairs, social, and educational activities; all of which could be a significant threat to child's survival. Eventually, the past decade has seen a paradigm shift in the study of disease origins (pathogenesis) and psychopathology towards positive psychology [10]. It has been increasingly recognised that many children and parents successfully negotiate salient developmental tasks inspite of major stressors such as acute or chronic illness and possible underlying emotional distress [11]. As noted in recent literature on other childhood chronic illnesses and disabilities [12], some positive implications of illness seen in this patient was normalization and the attribution of meaning. A central theme of psychological intervention was empowerment, which referred to providing the chronically ill child and her caregivers with the maximum amount of control over their own lives.

\section{CONCLUSION}

We may state that in order to achieve and sustain resilient adaptation, the child must receive support from adults in the environment along with his/her own personal attributes. Caring relationships, promoting effective coping and opportunities for meaningful participation proved quite important in child's capacity for innovative survival and ability to resist adversities.

$* * * * * * * * *$

\section{Author Contributions}

Manisha Jha - Substantial contributions to conception and design, Acquisition of data, analysis and interpretation of data, Drafting and revising article critically for important intellectual content; and Final approval of the version to be published

Ruchi Varma Shanker - Substantial contributions to conception, design and acquisition of data, Drafting the article for important intellectual content; and Final approval of the version to be published

Anand Kumar - Substantial contributions to conception and design, acquisition of data and interpretation of data, Drafting the article and revising it critically for important intellectual content; and Final approval of the version to be published

Tej Bahadur Singh - Substantial contributions to conception and design, Drafting the article for important intellectual content; and Final approval of the version to be published

\section{Guarantor}

The corresponding author is the guarantor of submission.

\section{Conflict of Interest}

Authors declare no conflict of interest.

\section{Copyright}

(C) Manisha Jha et al. 2013; This article is distributed under the terms of Creative Commons Attribution 3.0 License which permits unrestricted use, distribution and reproduction in any means provided the original authors and original publisher are properly credited. (Please see www.ijcasereportsandimages.com /copyright-policy.php for more information.)

\section{REFERENCES}

1. Wechsler D. The Wechsler intelligence scale for children-fourth edition. London: Pearson Assessment 2004.

2. Golden GJ. Luria-Nebraska Neuropsychological Battery Children's Revision (LNNB-C). Western Psychological Services, Torrance, CA 1987.

3. Wenck LS. House-Tree-Person Drawings: An Illustrated Diagnostic Handbook. Torrance, CA: Western Psychological Services 1970.

4. Rosenberg M. Rosenberg self-esteem scale: Society and The adolescent Self-Image. Princeton, N.J: Princeton University Press 1965.

5. Kovacs M. Children's Depression Inventory (CDI). New York: Multi-health Systems, Inc 1992.

6. Abidin RR. Parenting Stress Index manual (3rd ed) Charlottesville, V.A: Pediatric Psychology Press 1990.

7. Folkman S, Lazarus RS. Ways of Coping Ouestionnaire: (Research Ed.). Redwood City, CA: Consulting Psychologists Press 1988.

8. Veit CT, Ware JE Jr. The structure of psychological distress and well-being in general populations. Journal of Consulting and Clinical Psychology 1983:51(5):730-42.

9. Patterson JM. Promoting resilience in families experiencing stress. Pediatric Clinics of North America 1995;42(1):47-63. 
10. Linley PA. Positive psychology in practice. Hoboken, NJ: Wiley Press 1995.

11. Benzie K, Mychasiuk R. Fostering family resiliency: A review of the key protective factors. Child \& Family Social Work 2009;14(1):103-14.

12. Bayat M. Evidence of resilience in families of children with autism. Journal of Intellectual Disability Research 2007;51(Pt 9):702-14.

Access full text article on other devices

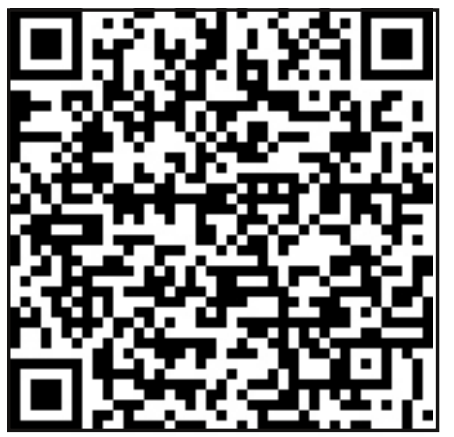

Access PDF of article on other devices

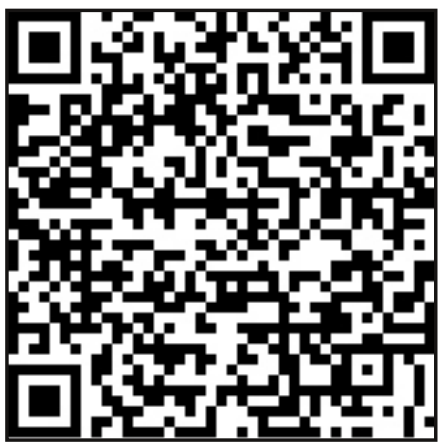

